\title{
Hinode XRT observations of a long-lasting coronal sigmoid
}

\author{
D. E. McKenzie and R. C. Canfield
}

\author{
Department of Physics, Montana State University, PO Box 173840, Bozeman, MT 59717-3840, USA \\ e-mail: mckenzie@solar.physics.montana.edu
}

Received 9 November 2007 / Accepted 28 December 2007

\section{ABSTRACT}

\begin{abstract}
Aims. Coronal sigmoids are important sources of eruptions into interplanetary space, and a handful of models have been proposed to explain their characteristic S shape. However, the coronal X-ray images available to date have generally not had sufficient resolution to distinguish between these models. The goal of the present investigation is to determine whether the new observations from Hinode can help us to make such a distinction.

Methods. We present the first observations of a persistent coronal sigmoid obtained with the Hinode X-Ray Telescope (XRT). The excellent angular resolution of XRT ( 1 arcsec per pixel) and the sigmoid's location near disk center combined to provide an unprecedented view of the formation and eruption of this phenomenon. We compared the observed morphology with expectations inferred from two popular models of sigmoid formation, the bald-patch separatrix surface model and the kinking flux rope model.

Results. The images during the pre-eruptive phase show that the overall S shape of the sigmoid comprises two separate J-shaped bundles of many loops. The straight sections of the two J patterns lie anti-parallel to one another in the middle of the S, on opposite sides of the magnetic polarity inversion line. The images during the eruptive phase reveal that, before any soft X-ray flaring begins, a diffuse linear structure almost as long as the sigmoid lifts off from the middle of the S. It shows slight clockwise rotation. The X-ray flare begins with the appearance of a sheared arcade of short loops, in the area centered between the two J-shaped patterns of the sigmoid.

Conclusions. Taken together, the observational findings provide strong support for the bald-patch separatrix surface model for this sigmoid.
\end{abstract}

Key words. Sun: corona - Sun: flares - Sun: magnetic fields

\section{Introduction}

The term "coronal sigmoid" refers to a coronal active region with an $\mathrm{S}$ or reversed-S shape as viewed in X-rays. The term was coined by Rust \& Kumar (1996) in describing transient S-shaped brightenings immediately preceding CME eruptions. The telltale signature of these eruptions is the "sigmoid to arcade" evolution (cf. Sterling \& Hudson 1997) as demonstrated in Fig. 1 of Rust \& Kumar (1996). The particular case shown in that figure is an example of a so-called "transient sigmoid", becoming clearly noticeable only a short time before the actual eruption. This contrasts with longer-lasting sigmoids, which display the $\mathrm{S}$ shape for many hours or even several days. Such persistent sigmoids hold their shape and appearance right up until the eventual eruption, sometimes becoming more clearly $\mathrm{S}$-shaped in the last few hours before the eruption. The current paper discusses such a persistent sigmoid, observed over the span of about 3 days.

In a 1999 study, Canfield, Hudson, and McKenzie examined 2 years' worth of full-Sun images obtained with the Yohkoh Soft $\mathrm{X}$-ray Telescope (SXT), identifying arcades and cusped loops as observational signatures of eruptive activity. More than $100 \mathrm{ac}-$ tive regions were categorized according to whether they were sigmoidal or non-sigmoidal, and also according to whether any eruptive signature was found. The conclusion was that "those [active regions] with sigmoidal morphology, regardless of size, are $68 \%$ more likely to be eruptive than non-sigmoidal regions." More recently, Canfield et al. (2007) examined 107 sigmoidal active regions from the full span of the Yohkoh mission. Those authors identified 163 eruptions associated with the sigmoids, $83 \%$ of which were detected as CMEs by LASCO.

With such a foundation in the evidence showing the relevance of sigmoids to solar activity and space weather, the motivation to understand their structure and evolution is clear. The Canfield et al. (1999) and Canfield et al. (2007) results have demonstrated that sigmoids are frequently associated with CMEs, but no signature has been identified to allow prediction of when an eruption might occur. It seems reasonable to suppose that a theoretical model that explains the configuration of a sigmoid's magnetic fields would enhance our understanding of the stresses imposed on/by those constituent fields. Such an improved understanding would facilitate estimating the likelihood of a potentially geoeffective eruption. Although a number of models have been proffered over the years to describe these special active regions, distinguishing observationally between competing explanations for the shape of sigmoids has been difficult. The goal of the present investigation is to determine whether the new observations from Hinode can help us to make such a distinction.

We present in this paper analysis of the first long-lasting coronal sigmoid observed with the X-Ray Telescope (XRT) on board Hinode (Kosugi et al. 2007; Golub et al. 2007). The angular resolution and sensitivity allow an unprecedented view into the detailed makeup of this pre-eruptive structure. We begin with a brief synopsis of two magnetic models of sigmoids. Next we introduce the XRT observations, and then compare the images directly to the predictions of the theoretical models. This is done in two parts: first in regards to the pre-eruption observations, and 


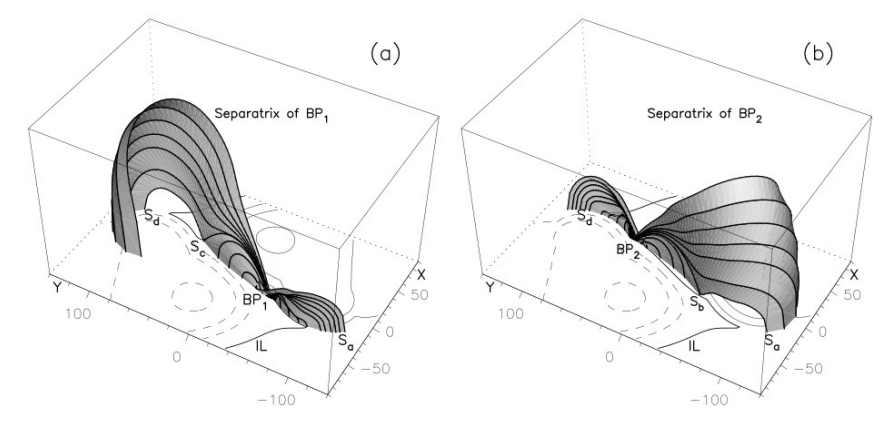

Fig. 1. Separatrix surfaces associated with bifurcated bald patches resemble the letter "J" in projection (reproduced from Titov \& Demoulin 1999).

secondly with regards to the observed eruption. We will see that the data help to distinguish between the two different models of sigmoid formation.

\section{Models of sigmoid shape}

Essentially all of the models that have been put forth involve twisted ropes of magnetic flux, since the twist affords a convenient method of storing energy needed for an eruption, and the appearance of the sigmoids is suggestive of writhing coronal loops. For the present study, we focus on two widely invoked models, both of which consider a twisted flux rope embedded within some ambient magnetic field.

\subsection{Bald-patch separatrix surface model}

Titov \& Demoulin (1999) considered a static flux rope embedded in a pre-existing field and recognize that there are places where the magnetic field wrapped around the axis of the flux rope would be tangential to the photosphere and concave upwards. Such an orientation is referred to as a "bald patch", and Titov \& Demoulin (1999) identified separatrix surfaces associated with the bald patches formed during flux rope emergence. Particularly after the bald patch bifurcates, two separatrix surfaces are present (see Fig. 7 of Titov \& Demoulin 1999). A separatrix defines the boundary surface between two different domains where the magnetic field changes connectivity. Field lines in the separatrix surface have footpoints in the bald patch, near the polarity inversion line. When applied to sigmoids, the bright coronal loops that make up the sigmoid are interpreted as field lines in or near the separatrix surface. When seen from above, i.e., when projected onto the plane of the sky, these surfaces resemble the letter "J" (see Fig. 1, reproduced from Titov \& Demoulin 1999). A J-shaped separatrix surface is associated with each of the two bald patches, so that the overall appearance is that of two J's wrapping around each other along the polarity inversion line. When viewed together the overall shape of the collection of loops in the active region is the familiar $S$ of the sigmoid. This "2-J" configuration is a key prediction of the bald-patch separatrix surface (BPSS) model.

\subsection{Kinking flux rope model}

Fan \& Gibson (2004) performed numerical MHD simulations of a kinking flux rope surrounded by an ambient magnetic field. They find that the field lines of the kinking flux rope have a significantly different orientation than the neighboring ambient field lines, so tangential discountinuities will exist at the interface between the kinking flux rope and the ambient field, specifically surrounding the "legs" of the kinking rope (see Figs. 2 and 5 of Fan \& Gibson 2004). These tangential discontinuities imply the presence of a current sheet in the corona; in fact, the shape of the predicted current sheet is S- or reverse-Sshaped, depending on the handedness of the kinking flux rope. Figure 5 of Fan \& Gibson (2004) shows a slice through the current sheet at a quasi-arbitrary height - the most intense current is located in an S-shaped sheet that is smooth and continuous. This is the essence of the model's prediction of sigmoid shape: a dynamic S-shaped sheet of intense current density. If the plasma located in/near this sheet becomes heated, perhaps by the current itself or perhaps by reconnection across the current sheet, then one may expect to see an S-shaped locus of heated plasma, i.e., a coronal sigmoid.

\section{XRT observations}

A sigmoid was observed on the Sun from in mid-February 2007, and XRT made images with its full resolution of 1 arcsec per pixel. Over the interval 09 Feb., 10:51 UT to 12 Feb., 05:30 UT, the image cadence was typically one image per $30 \mathrm{~s}$, with occasional bursts of one image per $10 \mathrm{~s}$. Because the polar orbit of Hinode affords a continuous view of the Sun for several months each year, the data coverage during this interval is remarkably complete. There is a 10-h gap between 10 Feb., 17:56 UT and 11 Feb., 06:11 UT, and a few gaps on the order of $10 \mathrm{~min}$ each, early on $12 \mathrm{Feb}$. The field of view for the high-cadence images was $384^{\prime \prime} \times 384^{\prime \prime}$. From 12 Feb., 05:30 UT onward, including the eruption of the sigmoid, we extracted the relevant field of view from full-Sun images, with the same angular resolution and a cadence of one image per $20 \mathrm{~min}$. The primary filter for the sigmoid observations was the "thin-aluminum/polyimide" (or "Al/poly") filter, imaging plasmas with temperature of roughly $2-5 \mathrm{MK}$ in the active region. Occasional images in the "titanium/polyimide" ("Ti/poly") filter were also made every $12-15 \mathrm{~min}$. For the present study, only the Al-poly images were utilized.

From the first images on 09 Feb., the active region's shape is suggestive of a sigmoid. The characteristic $\mathrm{S}$ shape is clearly distinguishable by 11:00 UT on 10 Feb., and becomes progressively more distinct until the sigmoid's eruption at roughly 06:21 UT on 12 Feb. From Fig. 2, taken at 07:59 UT on 11 Feb., one can see that the sigmoid comprises many loops/strands extending along its length. This is consistent with the findings of Canfield et al. (2007). An important feature of these many loops/strands is that they appear to define two J-shaped bundles. We will discuss this below.

Notably, the sigmoid appears to be completely disrupted by the eruption. By 00:00 UT on 13 Feb., the post-eruption arcade has faded; thereafter, there is no apparent trace of the S shape, and indeed the active region as a whole fades almost entirely from view over the next $24 \mathrm{~h}$.

\section{Discussion}

In comparison with the predictions of sigmoid shape from the two models considered here, we recall that the BPSS model includes the possibility of two J-shaped collections of loops aligned with the separatrices, whereas the kinking flux rope (KFR) model indicates a transient, smooth, S-shaped current sheet crossing the polarity inversion line underneath the erupting flux rope. In Fig. 2, the coronal loops appear to assume J-shaped 


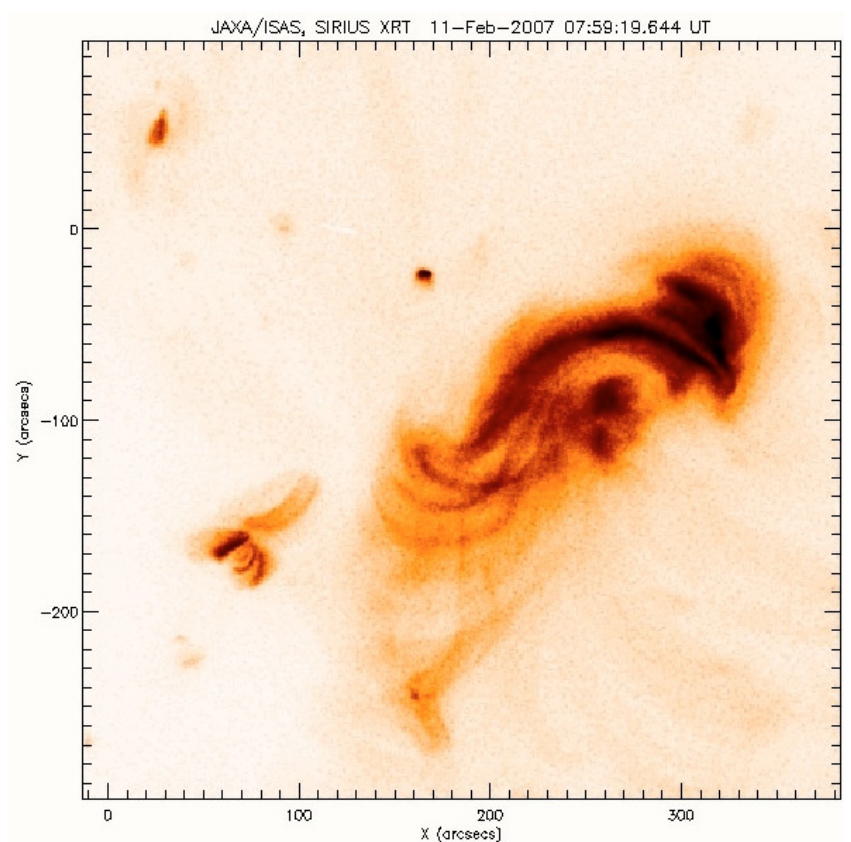

Fig. 2. XRT image of the coronal sigmoid at 07:59 UT on 11 Feb. 2007. The sigmoid comprises many individual loops, in two J-shaped collectives.

bundles. This is seen also in Fig. 3, where contours of X-ray brightness are overlaid on a line-of-sight magnetogram from $\mathrm{SOHO} / \mathrm{MDI}$. Because the northern J is significantly brighter than the southern J, it was difficult to find a single set of contour levels for this image that would outline both J's simultaneously. We therefore selected the loops of each $\mathrm{J}$ and plotted a brightness contour at a signal level that was appropriate for each. Examination of Fig. 3 suggests that the "straight" ends of the J-shaped bundles terminate in low-field-strength regions near the polarity inversion line. In the BPSS model, these footpoints correspond to the bald patches.

We also note that the KFR model predicts the intense S-shaped current sheet specifically during the time when the flux rope is in motion. The model requires motion of the rotating flux rope. In the simulations of Fan \& Gibson, the current sheet persists for 10-40 Alfvén crossing times; given the parameters of that simulation, the timescales of interest are 20-60 min. In contrast, the BPSS model allows the J-shaped separatrices at any time after the formation of the bald patches. No motion is required for the appearance of a sigmoid in the BPSS model, so in principle a BPSS sigmoid can persist for any arbitrary time unless/until the flux rope happens to erupt. This is relevant to the present discussion because the active region held its sigmoid shape for at least 2 full days before erupting. As mentioned above, the structure was discernable as a two-J sigmoid from at least $10 \mathrm{Feb} ., 11: 00 \mathrm{UT}$ (and possibly $24 \mathrm{~h}$ earlier, though the shape is harder to define at that time). The sigmoid erupted on 12 Feb., at 07:00 UT. This time span is obviously much longer than the 1-h timescale implied by the KFR model.

When this sigmoid erupted on 12 Feb., a bright bar-shape feature was observed rising from the central part of the sigmoid (Fig. 4). Because of its location there, its length (nearly as long as the central axis), and the fact that it appears to rotate slightly as it rises, we speculate that this bar-shaped feature may actually be the flux rope itself, in the act of erupting. The clockwise direction of rotation of the bar-shaped feature is consistent with

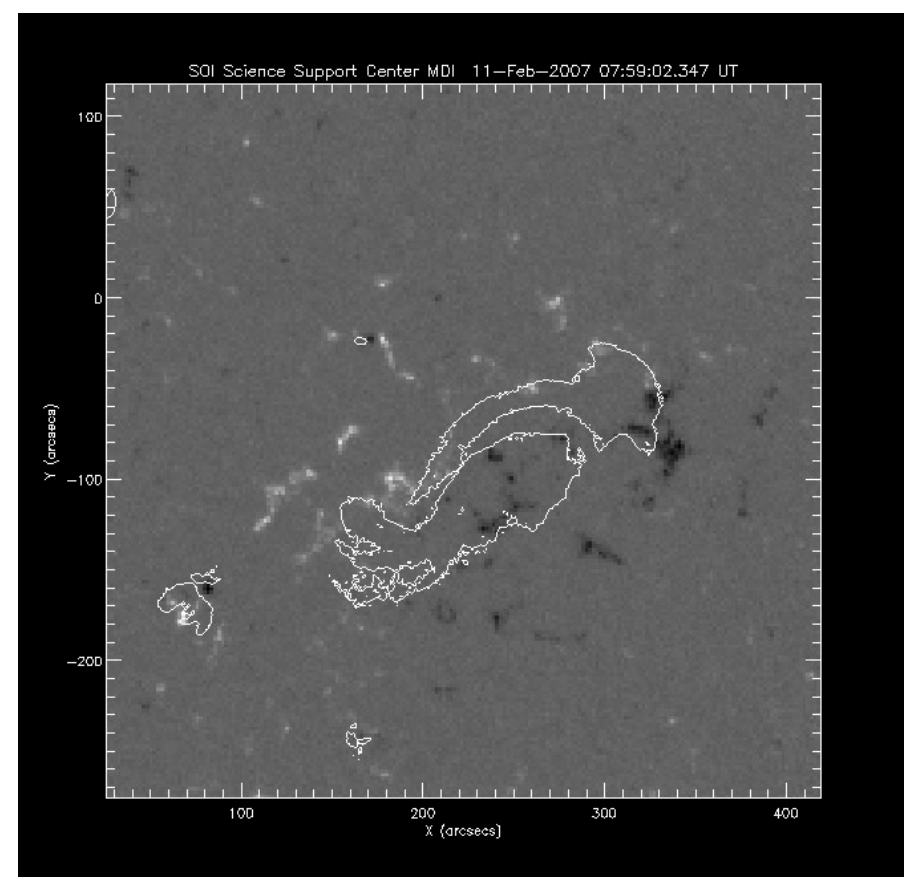

Fig. 3. Comparison of the XRT sigmoid image (contours) with the lineof-sight magnetogram from SOHO/MDI.

expectations of a kinking flux rope: to conserve magnetic helicity, the writhing of the flux rope is expected to keep the same sense as the twist in the field lines. The sigmoid in the present observations has a right-handed twist. A right- (left-)handed flux rope, when kinking, should rotate clockwise (counterclockwise). In the present case, the rotation was clockwise as expected from kinking, but slow (only about 10 degrees in the span of $40 \mathrm{~min}$ ). The identification of the rotating bar-shaped feature with a kinking flux rope is thus speculative, but the direction of the rotation and the circumstances of the feature's appearance are consistent.

The sigmoid's eruption shows a key element of the BPSS model. The separator field line fulfills the role of the $x$-point in the standard 2-D model of reconnection in flares, and forms the spine of the post-eruption arcade along the polarity inversion line. As seen in Fig. 4, the first sign of the cusped arcade appears precisely underneath the rising bar-shaped feature, along the polarity inversion line. This later grows into the full-fledged post-eruption X-ray arcade (not shown).

The subsequent fading of the active region, seeming to disappear from the Sun's face, would suggest that the flux rope erupted completely in this specific case, although this interpretation is speculative.

\section{Summary}

Although one might expect that the higher angular resolution of TRACE ( 0.5 arcsec per pixel) would allow a more detailed view of the internal structure of sigmoids, this has generally not been the case. From several studies (Sterling et al. 2000; Gibson et al. 2002; Liu et al. 2007; Green et al. 2007), it is known that TRACE and similar EUV imagers typically only detect the sigmoid shape 1-4 h before eruption; i.e., TRACE typically only sees "transient" sigmoids. As explained by Gibson et al. (2002), based on comparison of SXT, CDS, and TRACE data, the reason appears to be that, prior to the pre-eruption activation of the sigmoid, very little of the $1 \mathrm{MK}$ plasma visible to TRACE is present. Thus, TRACE observatons have been more useful for 

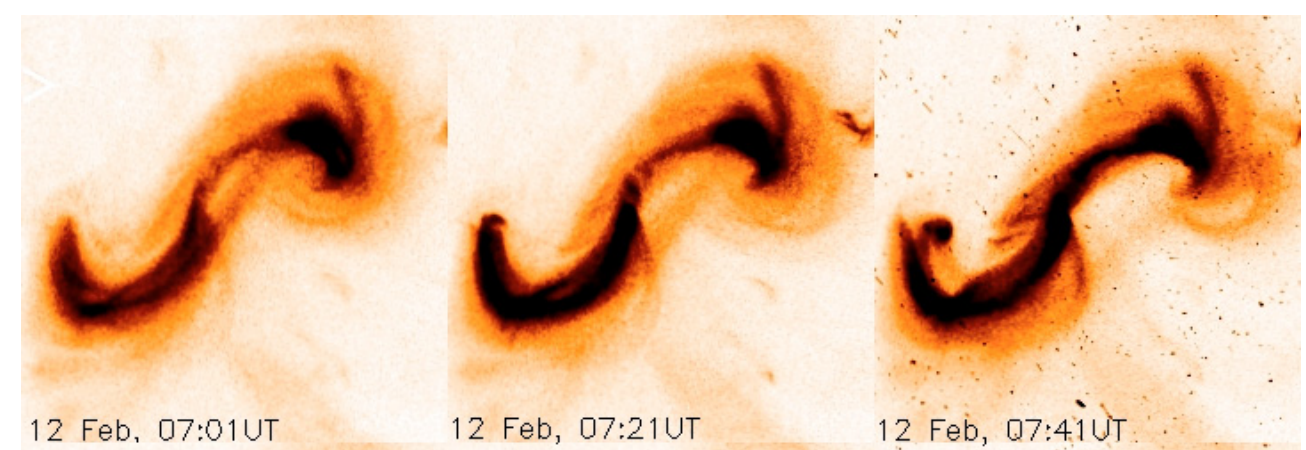

12 Feb, 07:01UT

12 Feb, 07:21UT

12 Feb, 07:410广
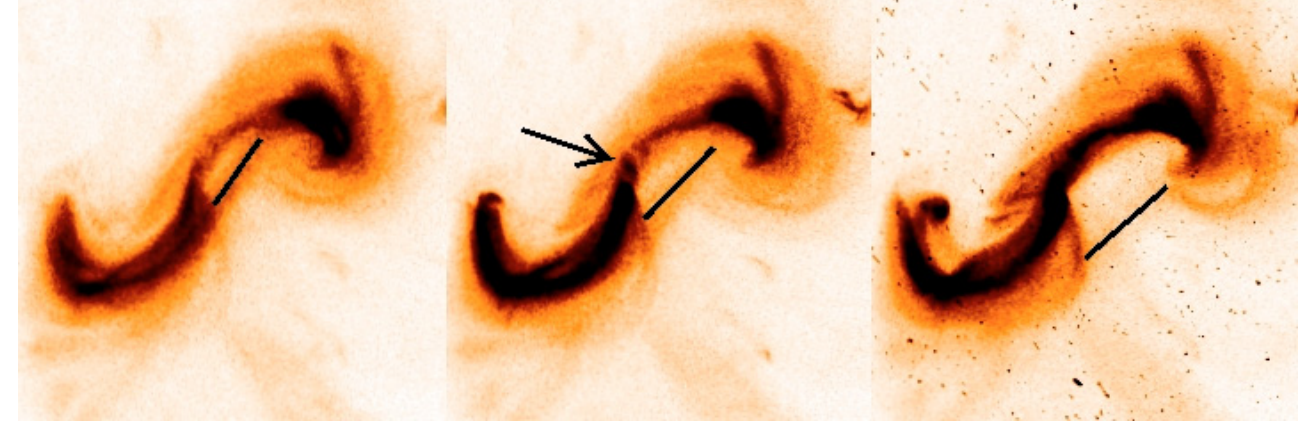

Fig. 4. XRT images of the bar-shaped feature rising above the erupting sigmoid. The lower three panels represent the same times as the top three, and include a tracer line as an aid to locating the bar-shaped feature. The arrow at 07:21 UT indicates the first appearance of the post-eruption arcade.

studying the activation of the sigmoid immediately preceding the eruption (cf. Liu et al. 2007) than for comparison with models explaining the shape of long-lasting sigmoids. In the present case, XRT's angular resolution (1 arcsec/pixel) and the sigmoid's location near disk center combined to provide an unprecedented view of the formation and eruption of this phenomenon. XRT observed the sigmoid over a 66-h period with a cadence of at least 1 image per $30 \mathrm{~s}$ (except for a 10-h gap), and with a much lower cadence for a few days prior. The first motions associated with eruption of the sigmoid started at 06:21 UT on 12 Feb. 2007; the first brightening of the ensuing X-ray arcade was seen at 07:21 UT.

The images during the pre-eruptive phase, which ends with the onset of large-scale motions, show:

- The overall S shape of the sigmoid is not defined by any single X-ray loop. Rather, many individual loops collectively create an S-shaped pattern.

- The $S$ shape is comprised of two separate $\mathrm{J}$ shapes, whose straight sections lie anti-parallel to one another in the middle of the $\mathrm{S}$, on opposite sides of the magnetic polarity inversion line.

The images during the eruptive phase show:

- Approximately 80 min before any soft X-ray flaring begins, a diffuse linear structure, almost as long as the sigmoid, lifts off from the middle of the $\mathrm{S}$. It shows slight clockwise rotation.

- The X-ray flare begins with the appearance of a sheared arcade of short loops, in the area centered between the two J-shaped patterns of the sigmoid.

- Within $16 \mathrm{~h}$ after the start of the flare, no S shape remains.

Taken together, these features provide strong support for the BPSS model of sigmoids put forth by Titov \& Demoulin (1999). Specifically, the two-J appearance in X-ray images strongly resembles the separatrices depicted in Titov \& Demoulin (1999). Additionally, the 2- to 3-day duration of the sigmoid seems to be more consistent with the BPSS model, since the KFR model appears to require the flux rope to be in motion for the sigmoid to be visible, and thus predicts visibility for timescales on the order of one hour. The rotation of the bar-shaped ejected feature is consistent with the expected motion of a kinking flux rope, at least in regards to the direction of the rotation. The location of the posteruption arcade, particularly its initial appearance, matches the expectation of the BPSS model, in which the arcade should form underneath the separator, along the polarity inversion line. The disappearance of the sigmoid-indeed the whole active regionafter the eruption is consistent with a complete removal of the flux rope at the time of the eruption.

Acknowledgements. Hinode is a Japanese mission developed and launched by ISAS/JAXA, collaborating with NAOJ as a domestic partner and with NASA and STFC (UK) as international partners. Scientific operation of the Hinode mission is conducted by the Hinode science team organized at ISAS/JAXA. This team mainly consists of scientists from institutes in the partner countries. Support for the post-launch operation is provided by JAXA and NAOJ (Japan), STFC (UK), NASA, ESA, and NSC (Norway).

\section{References}

Canfield, R. C., Hudson, H. S., \& McKenzie, D. E. 1999, Geophys. Res. Lett., 26,627

Canfield, R. C., Kazachenko, M. D., Acton, L. W., et al. 2007, ApJ, 671, L81

Fan, Y., \& Gibson, S. E. 2004, ApJ, 609, 1123

Gibson, S. E., Fletcher, L., Del Zanna, G., et al. 2002, ApJ, 574, 1021

Golub, L., DeLuca, E., Austin, G., et al. 2007, Sol. Phys., 243, 63

Green, L. M., Kliem, B., Török, T., van Driel-Gesztelyi, L., \& Attrill, G. D. R. 2007, Sol. Phys., 246, 365

Kosugi, T., Matsuzaki, K., Sakao, T., et al. 2007, Sol. Phys., 243, 3

Liu, C., Lee, J., Yurchyshyn, V., et al. 2007, ApJ, 669, 1372

Rust, D. M., \& Kumar, A. 1996, ApJ, 464, 199

Sterling, A. C., \& Hudson, H. S. 1997, ApJ, 491, 55

Sterling, A. C., Hudson, H. S., Thompson, B. J., \& Zarro, D. M. 2000, ApJ, 532, 628

Titov, V. S., \& Demoulin, P. 1999, A\&A, 351, 707 